\title{
SOLVING A DYNAMIC COMBINATORIAL AUCTIONS PROBLEM BY A HYBRID METAHEURISTIC BASED ON A FUZZY DOMINANCE RELATION
}

\author{
Larbi Asli ${ }^{1, *}$, MÉziane AÏDER ${ }^{1}$ And El-GhazAli TAlbi ${ }^{2}$
}

\begin{abstract}
This paper introduces a bi-objective winner determination problem which is based on English auctions. Most models of combinatorial auctions (winner determination problem) do not allow the bidder to update his offer, due to the fact that these mechanisms are static. However in reality bidders are in rough competition while there is time for auction. In this work we give a mathematical formulation of the dynamic model of the bi-objective winner determination problem, where the objectives are: (i) maximization of the total income, (ii) maximization of the number of items sold. This problem is based on the English auction mechanism, which allows bidders to renew their bids until the end of the exercise period. Then the solution is proposed by giving an algorithm based on an hybridization of a metaheuristic with a fuzzy dominance relation. A numerical experimentation using this algorithm on simulated data gives rise to satisfactory results.
\end{abstract}

Mathematics Subject Classification. 90C27, 90C70, 90C59.

Received March 16, 2017. Accepted June 19, 2018.

\section{INTRODUCTION}

The study of combinatorial auctions problems is very important whether in theory or in practice. Several mathematical models are designed to describe and understand the topic, we can cite the integer programming for combinatorial auctions winner determination [1], the resolution of the problem of transportation contracts $[3,15]$, the use of the set packing problem to model the problem [8], and other relative works $[1,2,9,12-14,20,21]$. Combinatorial Auctions are an important interdisciplinary field combining issues from economics, game theory, optimization, and computer science.

The Combinatorial Auctions Problem (CAP) is defined as a set of items submitted to sale facing many buyers. For reasons of complementarity between items, every buyer will want to buy his own subset of items for which he provides an estimate. Because of the possible intersection between subsets, conflicts can arise between

Keywords. Multi-objective combinatorial auctions, winner determination problem, fuzzy dominance, exercise period, dynamic model.

1 LaROMaD, Fac. Maths, USTHB, Pb 32, 16111Bab Ezzouar, Algeria.

2 INRIA Lille - Nord Europe research centre, Parc Scientifique de la Haute-Borne, 40, avenue Halley - A, Park Plaza, 59650 Villeneuve d'Ascq, France.

*Corresponding author: El-ghazali.Talbi@lifl.fr 
buyers [2]. The objectives of the seller are various. The most important one is to maximize the total profit of the sale. To achieve that, he has to solve an NP-hard combinatorial optimization problem.

All existing combinatorial auctions models in the literature do not allow the bidder to update his offer. This is due to the fact that the mechanisms used are static. However, in reality, bidders are in competition while there is time left for the auction.

In this work an adequate dynamic formulation to replicate the English auction process is given. An English combinatorial auction is described by a number $m$ of items in limited quantities for each one, which are offered for sale to potential buyers. These buyers have a limited period of time and opportunity to update their bids.

Each bid is a combination of items, formulated as a vector of dimension $m+1$, which contains $m$ quantities of items desired by the bidder and the price offered for this bid. Knowing the bids of each other, an algorithm that will list the temporary winners at every moment will be developed, which leaves to the bidders the opportunity to respond accordingly.

The winner determination problem in its bi-objective version includes, in addition to maximizing the total profit of the auction, a second objective which is the liquidation of a maximum number of items sold in auction. The seller must optimize simultaneously both objectives, that are contradictory.

The multi-objective kind of this problem requires the non-existence of a single solution, but a set of efficient solutions. Several works on such problems are based on the classical Pareto efficient mechanisms for comparing pseudo-solutions. This principle generates therefore huge volume of calculations when the size of the problems is considerable [5,22].

Several forms derived from the Pareto dominance are used to try to appease the resolution process, such as A-dominance, lexicographical dominance, cone dominance $[4-7,18]$, etc.

Fuzzy dominance is one of the alternatives designed to overcome the difficulty of calculating Pareto's front. This principle, used for the first time in the field of multi-objective combinatorial optimization by Sakawa et al. [19], gives interesting results, since several authors have used it in various fields of optimization $[4,6,7,10$, $11,16-18]$.

We will hybridize this mechanism with the tabu search metaheuristic with random walk in order to reduce the computational cost.

The choice of the tabu method is motivated by the simplicity of its operation as well as the ease of its implementation. Indeed, it allows savings of time of resolution for the large programs, and escapes the local optima by the use of a concept of memory strategy.

This work focuses essentially on:

- The development of a new dynamic model describing the behavior of the auctioneers which gives them the opportunity to update their bids during the exercise period, and covers seller's goals.

- Building an efficient algorithm that will be called "FM-CAP" algorithm to solve this problem in an optimal manner. The problem is a winner determination problem (WDP), which is modeled as a bi-objective multidimensional knapsack problem.

- Fuzzy dominance will be used to improve the multi-objective resolution process.

\section{Dynamic BI-OBJECTIVE COMBinAtorial AUCTIONS MODEL}

Online auctions are very important since they allow to exchange goods and services without moving and without physical encounter. Customers can run their applications for products from any place in world. However the price is not the only requirement of the owners and the customers, the delivery time and the number of products sold are also essential factors in the procedure besides other factors. These considerations motivate the development of an adequate multi-objective model that can meet the demands of bidders and sellers. 


\subsection{Preliminaries}

The development of this new model requires the introduction of several concepts and notations which are given below:

$E_{j}: j$ th bidder;

$t_{l}$ : the time when a bidder $E_{j}$ launches his bid;

$\operatorname{prev}(t)$ : the time of the previous event just before the present one at $t$.

$S_{j}(t)$ : an $(n+1)$-vector containing $\left(A_{j}(t), c_{j}(t)\right)$, and representing the temporary offer of the bidder $E_{j}$ at time $t$ where;

$A_{j}$ : an $n$-vector of item quantity contained in $S_{j}$;

$c_{j}(t)$ : the value of the bid $S_{j}$ at time $t$;

$\beta_{i}$ : number of copies of the item $i$;

$\mathrm{LTG}_{t}$ : temporary list of winners at time $t$;

$\mathrm{LFG}_{T}$ : final list of winners;

NEVT: new event;

$\operatorname{LEC}_{j}(t)$ : list of bidders in conflict with $E_{j}$ at time $t$;

$Z(t)$ : temporary gain at time $t$.

In addition to the concepts already introduced, we also need the following definitions.

Definition 1.1. The exercise period, denoted $T$, is defined to correspond to the time interval $[0, T]$ associated to the auction in which bidders can launch their offers.

Definition 1.2. Temporary offer $S_{j}\left(a_{i j}, c_{j}\right)$ is the offer made by the bidder $E_{j}$ at time $t_{l} \in[0, T]$, each offer contains:

$a_{i j}(t)$ : the $n$-dimensional vector representing the quantities of items required by the bidder $E_{j}$;

$c_{j}(t)$ : the bid price.

This bid may be updated during the exercise period.

Definition 1.3. Temporary winners LTG is the temporary list of winning bidders at time $t_{l} \in[0, T]$, (this list may change during the exercise period $T$ ).

Definition 1.4. The conflict graph [2] is the graph where the vertices are the deals and edges connecting the deals that can not be selected simultaneously. This graph can detect the conflicting deals (i.e. deals that share at least one object).

\section{2. $\mathrm{WDP}_{t}$ mathematical model}

We present here a dynamic bi-objective mathematical model of combinatorial auctions (WDP). This problem belongs to the class of combinatorial optimization problems. The most important parameters of the model are the numbers of items $m$ (that will be contained in a vector $\beta$, the numbers of units for each component is $\beta_{i}$ ), and an exercise period $T$.

This model includes two objectives, the first objective is the maximization of the total profit obtained by the auction, and the second one is the maximization of the number of items sold.

This model reproduces the behavior of an English auction where each bidder has the right to update his bid during the exercise period.

The model is built in a dynamic way. It starts at $t=0$, once the first bidder proposes a first bid $S_{1}\left(c_{1}(t), a_{i 1}(t)\right)$. Each offer contains a list of items $A_{j}$ with corresponding prices $c_{j}$. At this moment, the problem will contain a single variable and be 1-dimensional.

So, and as long as other bidders come, the model is completed with the possibility that each offer previously given can be changed depending on the status of the auction. 
For reasons of adequacy of the problem, the sum of the winning bids must not exceed the number of available items.

Let the decision variables be:

$$
x_{j}=\left\{\begin{array}{l}
1, \text { if the bid of } E_{j} \text { is accepted, } \quad j=1, \ldots, n . \\
0, \text { otherwise }
\end{array}\right.
$$

The full model at time $t \in[0, T]$ is:

$$
\mathrm{WDP}_{t}\left\{\begin{aligned}
f_{t}^{1}(x) & =\max \sum_{j=1}^{k} c_{j}(t) x_{j} \\
f_{t}^{2}(x) & =\max \sum_{i=1}^{m} \sum_{j=1}^{k} a_{i j}(t) x_{j} \\
\sum_{j=1}^{k} a_{i j}(t) x_{j} \leq \beta_{i} \quad & i=1, \ldots, m \\
x_{j} & \in\{0,1\} \quad j=1, \ldots, k .
\end{aligned}\right.
$$

Upon completion of the exercise period $(t=T)$, the model will be final and contain $n$ bidders.

$$
\mathrm{WDP}_{T}\left\{\begin{aligned}
& f_{t}^{1}(x)=\max \quad \sum_{j=1}^{n} c_{j} x_{j} \\
& f_{t}^{2}(x)=\max \sum_{i=1}^{m} \sum_{j=1}^{n} a_{i j} x_{j} \\
& \sum_{j=1}^{n} a_{i j} x_{j} \leq \beta_{i} \quad i=1, \ldots, m \\
& x_{j} \in\{0,1\} j=1, \ldots, n .
\end{aligned}\right.
$$

\section{Resolution Algorithm For The $\mathrm{WDP}_{T}$ MODEL}

An algorithm that gives at each time of the exercise period the list of temporary potential winners is developed. This algorithm is designed in a way to escape if possible solving $\mathrm{WDP}_{t}$ problems. This is based on the analysis of bids at each new event. If there is no conflict with the temporary winning bids, the new bidder will also be winning. Otherwise, we resolve the $\mathrm{WDP}_{t}$ problem by the Metaheuristic random walk tabu search.

At the opening of the auction $(t=0)$, bidders launch their bids, the first bidder $E_{1}$ launches the bid $S_{1}$ at time $t_{1}$. It will be represented by a $(m+1)$-vector $S_{1}$, where each component $a_{1 j}$ contains the number of units requested for the article $\beta_{j}$. Thus, the value $c_{1}$ of the bidder $E_{1}$ will be considered a temporary winner.

At time $t_{2}$, a second bidder $E_{2}$ launches his submission $S_{2}$. If the second bid $S_{2}$ is not in conflict with the first bid $S_{1}$, the bidder $E_{2}$ will be also a temporary winner. Otherwise, if the bidder $E_{2}$ dominates the bidder $E_{1}$, then $E_{2}$ replaces $E_{1}$ in the list of temporary winners.

From this moment we can have a new bid $S_{j}$ or an update of a bid already made. The model is completed until the end of the exercise period $t=T$. Each event will be treated in a manner that will not lead us to solve the temporary problem $\mathrm{WDP}_{t}$.

The necessary ingredients for this algorithm are the tabu search metaheuristic, and the fuzzy logic dominance notion, that we will be explained. 


\subsection{Random Walk Tabu Search (RWTS) algorithm}

Contrary to the basic tabu search algorithm, where the diversification is ensured only by the tabu list, random walk tabu search algorithm consists of realizing sometimes a move which is no more guided by the evaluation function and then constitutes a diversification diagram.

At every iteration of the algorithm "RWTS", a real value $r w \in[0,1]$ is randomly generated. Let us put $q \in[0,1]$ the value threshold, then, if $r w>q$ the algorithm will select the best movement, otherwise, the algorithm will make a feasible random movement. The algorithm RWTS can be described as follows:

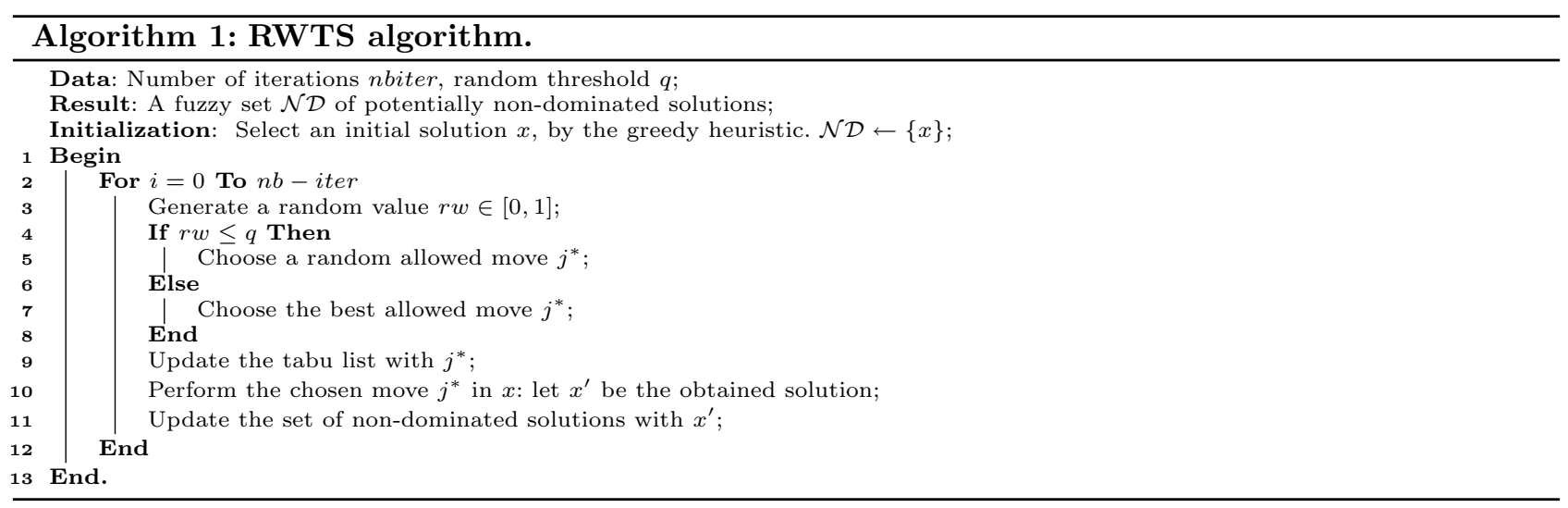

\subsection{Fuzzy dominance relation}

Due to the benefits and the efficiency in the resolution of optimization problems, fuzzy logic was become useful in several areas of optimization, like the combinatorial optimization with or without fuzzy parameters, the integer programming and nonlinear programming $[6,7,16,19]$.

Sakawa used fuzzy logic in several works, we can cite: Solving linear programming problems (0-1 programming) especially the multi-objective multi-dimensional knapsack problem and the multi-objective job shop problem [19]. He also used it in combinatorial optimization problems with fuzzy parameters. The concept is generalized for the integer multi-objective programming with fuzzy parameters. and he also used it in the nonlinear multiobjective programming with or without fuzzy parameters [19].

We also cite several uses of fuzzy dominance in the electricity domain as resolving a possibilistic model based planning of electrical distribution systems [18], or Electrical Discharge Machine using Fuzzy Evolutionary Strategies [6,10], etc.

Recent works in several areas of multi-objective optimization are focused on this concept, we cite goal programming [16], portfolio optimization [17], electricity distribution system planning [6], particle swarm optimization [4], as well as multi-objective workflow grid scheduling [7].

The multi-objective nature of CAP requires to use fuzzy logic at two levels of resolution; first, when comparing each new solution to the one already found in the non-dominated set $\mathcal{N} D$ (due to its very large size); second, when choosing a single solution at the end of the resolution process.

\section{Fuzzy dominance}

In this section we study the fuzzification of the Pareto dominance relation. We say that a vector $u$ Paretodominates a vector $v$ if $\left(\forall i \in\{1, \ldots, n\}\left(u_{i} \leq v_{i}\right)\right.$ with $\left.u_{j}<v_{j}\right)$ for at least one item $j \in\{1, \ldots, n\}$. Then the subset of all vectors which are not dominated by any other vector in the objective space $S$ is the Pareto 


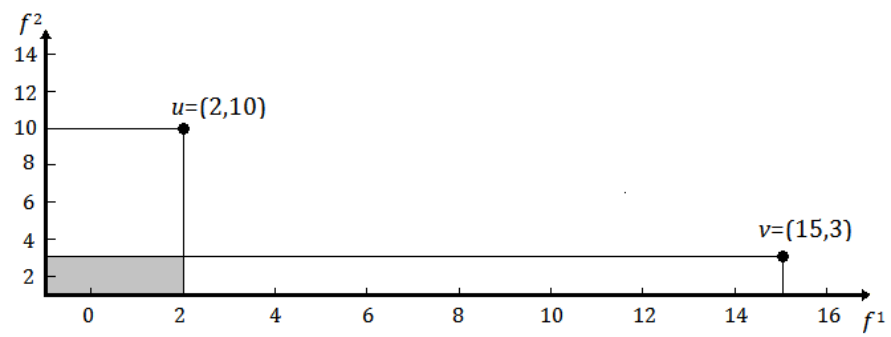

FiguRE 1. Fuzzy dominance between $u$ and $v$.

set. Note that in a similar manner, Pareto dominance can be related to the "> relation depending on the application context.

The interest of the Pareto front fuzzification is to provide for each pair of vectors a usable numerical representation of dominance. Fuzzy dominance is calculated once both the two following conditions are checked.

1. The measure is not symmetric and between two vectors $u$ and $v$, the two measures " $u$ dominates $v$ by degree $\mu_{a}$ " (active dominance), and " $u$ is dominated by $v$ with degree $\mu_{p}$ " (passive dominance) have to be distinguished, since the calculations of the two measures differ by the denominator and are therefore not symmetrical.

2. The dominance degrees are set-dependent and can not be assigned in an absolute manner to single vectors alone.

The fuzzy classification system for a generic set of vectors $S$ is based on the computation of the comparison value for each pair of vectors $\{u, v\}$ by using Definition 2.1, and then sort out the values in increasing or decreasing order depending on the optimization context.

Definition 2.1. [11] The fuzzification of Pareto dominance relation can be written as follows: A vector $u$ dominates a vector $v$ by degree $\mu_{a}$ with a value

$$
\mu_{a}(u, v)=\frac{\prod_{i} \min \left(u_{i}, v_{i}\right)}{\prod_{i} u_{i}}
$$

and that vector $u$ is dominated by vector $v$ with degree $\mu_{p}$ with

$$
\mu_{p}(u, v)=\frac{\prod_{i} \min \left(u_{i}, v_{i}\right)}{\prod_{i} v_{i}}
$$

Then we have:

$$
\mid \begin{aligned}
& \mu_{a}(u, v)=1, \text { if } u \text { Pareto-dominates } v \\
& \mu_{p}(u, v)=1, \text { if } v \text { Pareto-dominates } u \\
& \mu_{a}(u, v)<1, \text { otherwise. }
\end{aligned}
$$

Figure 1 gives a numerical example for the fuzzy Pareto dominance considered here between two vectors $u$ and $v$. Note that, when we compute $\mu$, if an item $u_{i}=0$ or $v_{i}=0$, this is handled by the exclusion in the products in the both numerator and denominator. Here, $u$ dominates $v$ by degree $\frac{2 \times 3}{2 \times 10}=0.3$ and is dominated by $v$ by degree $\frac{2 \times 3}{3 \times 15}=0.13$. 


\subsection{Fuzzy Multi-objective CAP-algorithm (FM-CAP)}

The steps of this algorithm are given by:

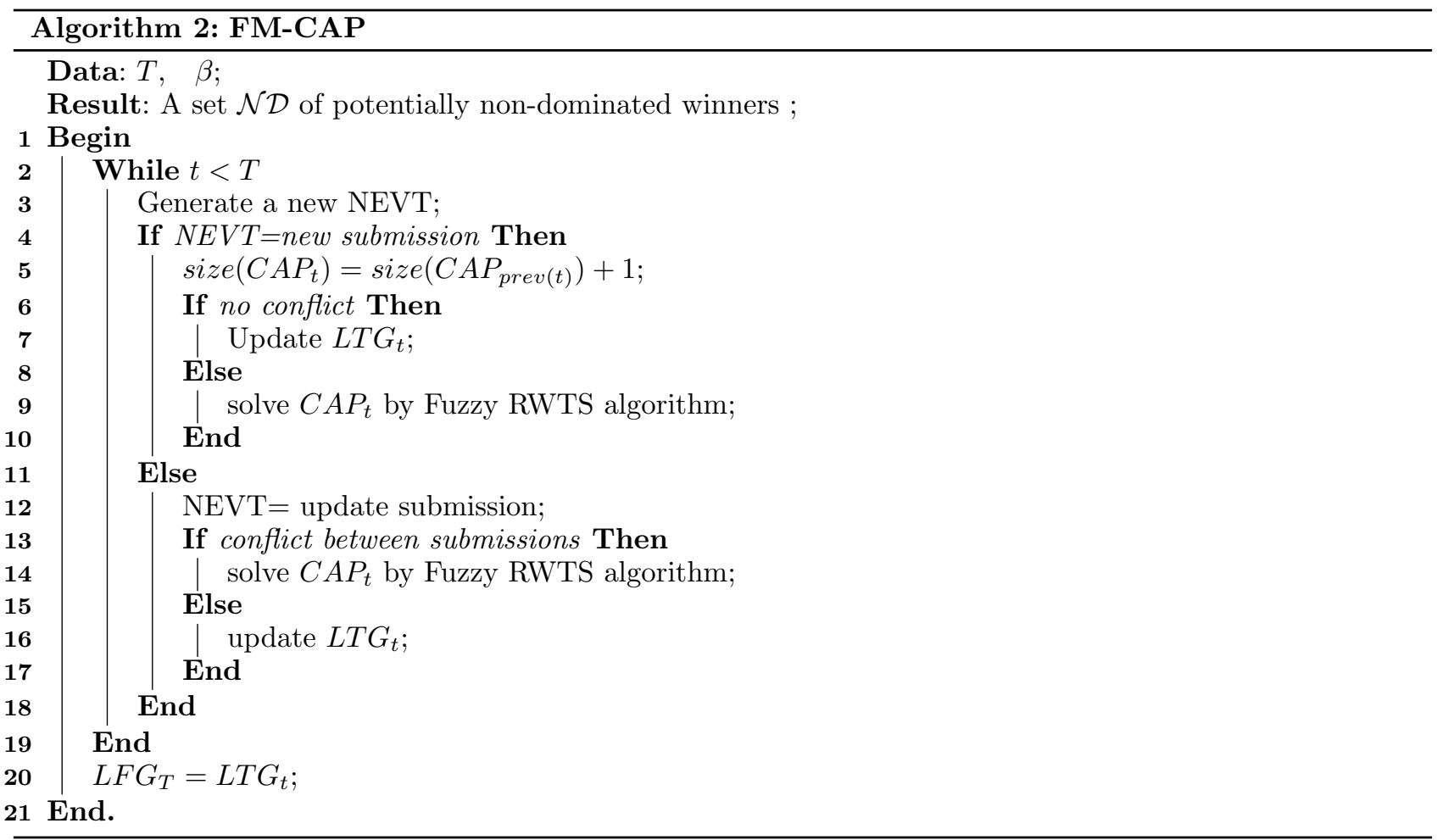

\section{Didactic EXAmple}

To better understand the behavior of this model and its solution algorithm, an illustration by a didactic example (numerical) is made.

A set of three products on sale, that includes for each one, the amounts given in the vector $\beta=(10,7,9)$ respectively, and an exercise period $T=10$ units of time.

During this period, four bidders will participate at the auction. They will express their bids as the quantities of items they need and the corresponding prices. During the period $T$, these four bidders will give their bids and will update them. The progress of the operation is shown in the Table 1.

\subsection{Details on the calculation steps}

At time $t_{1}$, the first bidder $E_{1}$ launches his first bid $a_{1}^{t}=(2,3,5)$ for an estimate $c_{1}=15$. The temporary problem is:

$$
\mathrm{WDP}_{t_{1}}\left\{\begin{array}{cc}
f_{t}^{1}(x)=\max & 15 x_{1} \\
f_{t}^{2}(x)=\max & 2 x_{1}+3 x_{1}+5 x_{1} \\
& \left(\begin{array}{l}
2 \\
3 \\
5
\end{array}\right) \\
& x_{1} \leq\left(\begin{array}{c}
10 \\
7 \\
9
\end{array}\right) \\
& x_{1} \in\{0,1\}
\end{array}\right.
$$


L. ASLI ET AL.

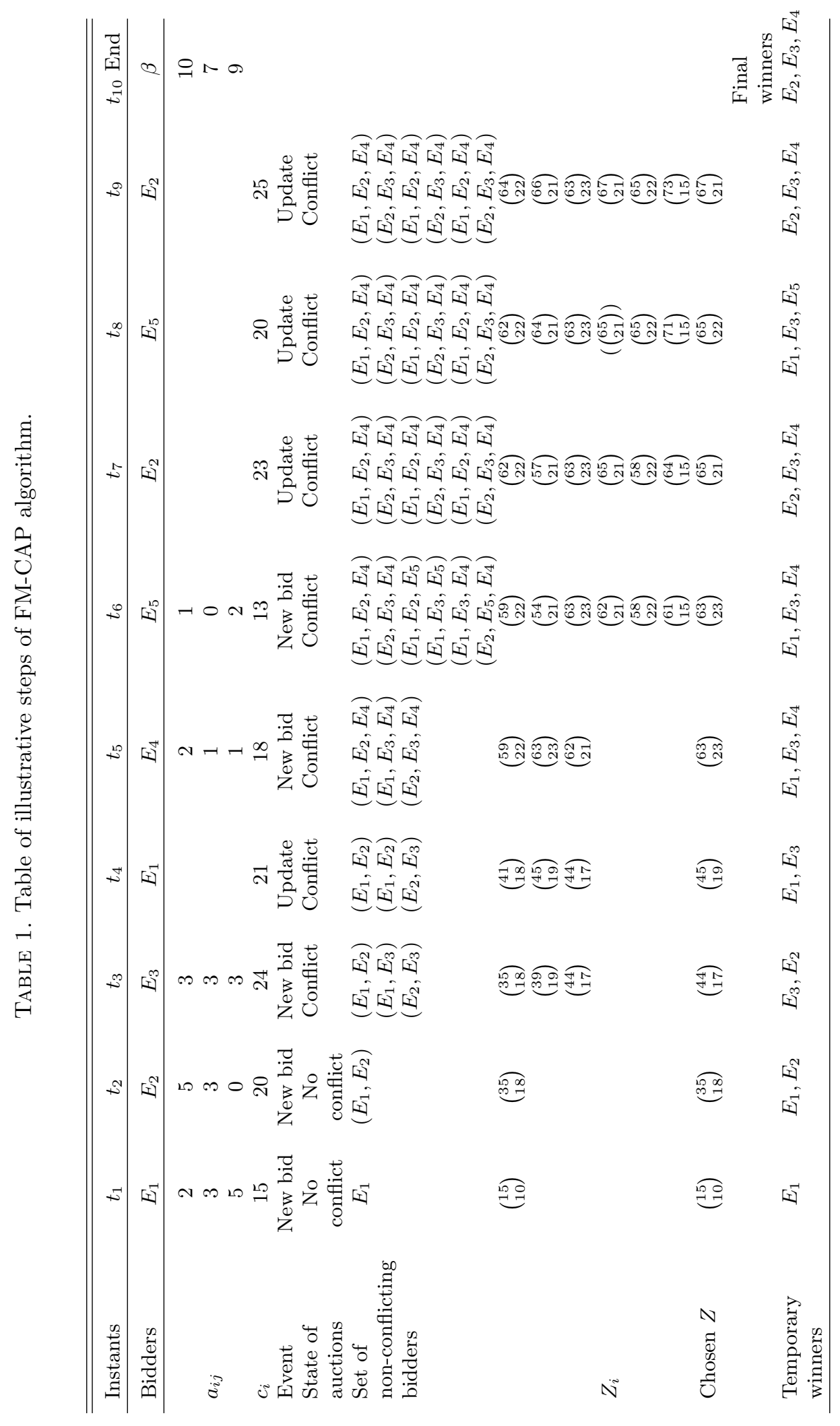


This event will be considered as a new bid. One can observe that there is no conflict, because $E_{1}$ is alone in this auction at this time, therefore he will be a first temporary winner. The objective function value is:

$$
Z_{1}=\left(\begin{array}{c}
15 \\
2+3+5
\end{array}\right)=\left(\begin{array}{l}
15 \\
10
\end{array}\right)
$$

A new bid is made at time $t_{2}$ by the bidder $E_{2}$ containing a bid $a_{2}^{t}=(5,3,0)$ with $c_{2}=20$. The problem has two bidders:

$$
\mathrm{WDP}_{t_{2}}\left\{\begin{array}{rr}
f_{t}^{1}(x)=\max & (15,20)\left(\begin{array}{l}
x_{1} \\
x_{2}
\end{array}\right) \\
f_{t}^{2}(x)=\max & (2,5)\left(\begin{array}{l}
x_{1} \\
x_{2}
\end{array}\right)+(3,3)\left(\begin{array}{l}
x_{1} \\
x_{2}
\end{array}\right)+(5,0)\left(\begin{array}{l}
x_{1} \\
x_{2}
\end{array}\right) \\
\left(\begin{array}{ll}
2 & 5 \\
3 & 3 \\
5 & 0
\end{array}\right)\left(\begin{array}{l}
x_{1} \\
x_{2}
\end{array}\right) \leq\left(\begin{array}{c}
10 \\
7 \\
9
\end{array}\right) \\
x \in\{0,1\}^{2}
\end{array}\right.
$$

This bid does not present any conflict with $E_{1}$. Then it will also become temporary winner with objective function value:

$$
Z_{2}=\left(\begin{array}{c}
15+20 \\
(2+5)+(3+3)+(5+0)
\end{array}\right)=\left(\begin{array}{l}
35 \\
18
\end{array}\right)
$$

No change for the temporary list until $t_{3}$, when $E_{3}$ enters to the game, so the problem evolves as follows:

$$
\operatorname{WDP}_{t_{3}}\left\{\begin{array}{rr}
f_{t}^{1}(x)=\max & (15,20,24)\left(\begin{array}{l}
x_{1} \\
x_{2} \\
x_{3}
\end{array}\right) \\
f_{t}^{2}(x)=\max & (2,5,3)\left(\begin{array}{l}
x_{1} \\
x_{2} \\
x_{3}
\end{array}\right)+(3,3,3)\left(\begin{array}{l}
x_{1} \\
x_{2} \\
x_{3}
\end{array}\right)+(5,0,3)\left(\begin{array}{l}
x_{1} \\
x_{2} \\
x_{3}
\end{array}\right) \\
\left(\begin{array}{lll}
2 & 5 & 3 \\
3 & 3 & 3 \\
5 & 0 & 3
\end{array}\right)\left(\begin{array}{l}
x_{1} \\
x_{2} \\
x_{3}
\end{array}\right) \leq\left(\begin{array}{c}
10 \\
7 \\
9
\end{array}\right) \\
x \in\{0,1\}^{3}
\end{array}\right.
$$

The bid of $E_{3}$ compounds $a_{3 j}=(3,3,3)$ with $c_{3}=24$ and this makes a conflict with both $E_{1}$ and $E_{2}$. At this moment, the set of solutions is:

$$
Z_{3}=\left\{Z_{31}=\left(\begin{array}{l}
35 \\
18
\end{array}\right), Z_{32}=\left(\begin{array}{c}
39 \\
19
\end{array}\right), Z_{33}=\left(\begin{array}{l}
44 \\
17
\end{array}\right)\right\}
$$

But $Z_{31}$ is dominated by $Z_{32}$, therefore, the set of effective solutions is $\left\{Z_{32}=\left(\begin{array}{l}39 \\ 19\end{array}\right), Z_{33}=\left(\begin{array}{c}44 \\ 17\end{array}\right)\right\}$ which represents the winning couples $\left(E_{1}, E_{3}\right),\left(E_{2}, E_{3}\right)$. We must choose one solution, we do that by fuzzy dominance:

$$
\left\{\begin{array}{l}
\mu_{a}\left(Z_{32}, Z_{33}\right)=\frac{\prod_{i} \min \left(u_{i}, v_{i}\right)}{\prod_{i} u_{i}}=\frac{19 \times 17}{39 \times 19}=0,435 \\
\mu_{p}\left(Z_{32}, Z_{33}\right)=\frac{\prod_{i} \min \left(u_{i}, v_{i}\right)}{\prod_{i} v_{i}}=\frac{19 \times 17}{44 \times 17}=0,431
\end{array}\right.
$$


The couple $\left\{E_{3}, E_{2}\right\}$ has the best evaluation, so $E_{3}$ and $E_{2}$ become temporary winners.

$E_{1}$ reacts by updating his price to $c_{1}=21$ at time $t_{4}$ and the same process at $t_{3}$ is made, $E_{1}$ rejoins $E_{3}$ as temporary winner.

From this moment, until $t=10$ units of time, the auction continues by application of the FM-CAP algorithm.

\section{NumericAl EXPERIMENTATION}

In order to evaluate the effectiveness of the fuzzy dominance mechanism, we proceed in this numerical part, to the implementation of the tabu search metaheuristic. Then we generate different size test problems for the bi-objective winner determination problem model, in order to compare the results obtained by the use of the two dominance mechanisms "Pareto and fuzzy". This comparison will build in addition of running time, on the calculation of the hypervolume metric which will be defined further.

These executions of tabu search metaheuristic (which is a part of the FM-CAP algorithm) consist of:

- Finding the appropriate parameters of the metaheuristic (the number of iterations and the threshold of the random walk).

- Discovering the mutual influences between the number of iterations, the hypervolume, the CPU time and the Pareto front size.

- In the end, executions of large instances of WDP problem to see the ability of the tabu method.

\subsection{Hyervolume}

This metric was introduced by Zitzler [22] to calculate an approximate volume under the curve formed by the points of the set being evaluated. Thus, when the problem has two criteria, this calculation is the calculation of an area. When the problem has three criteria, the calculated value is a volume, etc. The definition presented in Zitzler [22] is as follows:

Definition 4.1. Let $A=\left(x_{1}, x_{2}, \ldots, x_{n}\right) \subseteq X$ be a subset of $n$ elements. The $H$ function calculates the volume bounded by the union of all polytopes $p_{1}, p_{2}, \ldots, p_{n}$, where each $p_{i}$ is formed by the intersection of hyperplanes of $x_{i}$ with respect to the coordinate axes: for each axis of the objective space, there is a hyperplane perpendicular to the axis and passing through the point $\left(f_{1}\left(x_{i}\right), f_{2}\left(x_{i}\right), \ldots, f_{k}\left(x_{i}\right)\right)$. For the two-dimensional case, each $p_{i}$ represents the rectangle defined by the coordinate points $(0,0)$ and $\left(f_{1}\left(x_{i}\right), f_{2}\left(x_{i}\right)\right)$.

In the case of minimization problems where one seeks to minimize the values of objectives, and where compromise surfaces have the same number of points, the smaller the value of hypervolume is, the better is the compromise surface.

\subsection{Results}

We implement the programs on testing problems crafted such kinds to replicate some natural bidders behaviors, such:

- Strong individual request of bid;

- An average demand balanced among all the bidders;

- Weak demand.

Each instance of the problem is simulated based on the normal distibution, of fixed standard deviation $\sigma$ and average $\lambda$, which varies from 0 to $\min \beta_{i}$ depending on the case: a high average for the consequent requests, a low average for demands of small cardinalities and finally an average for ordinary applications.

All these executions are carried out on a machine with the following characteristics: windows 7, 64-bit, Intel(R)Core(TM) i3-2377M CPU 1,50 GHz, RAM 4,00 Go. 
TABLE 2. The influence of random walk on the tabu method.

\begin{tabular}{ccccccc}
\hline \hline RW & \multicolumn{2}{c}{ Cpu } & \multicolumn{2}{c}{ Size efficient solutions } & \multicolumn{2}{c}{ Hypervolume } \\
\hline & Fuzzy tabou & Pareto tabou & Fuzzy tabou & Pareto tabou & Fuzzy tabou & Pareto tabou \\
0,1 & 4.132309 & 4.169697 & 6 & 2 & 737962 & 541099 \\
0,2 & 4.247215 & 4.246543 & 4 & 2 & 672244 & 423298 \\
0,3 & 4.229345 & 4.235883 & 4 & 1 & 886936 & 302176 \\
0,4 & 4.273540 & 4.171221 & 4 & 3 & 751377 & 617758 \\
0,5 & 4.170811 & 4.097720 & 4 & 1 & 580488 & 234030 \\
0,6 & 4.270917 & 4.112718 & 4 & 1 & 884720 & 281428 \\
0,7 & 4.066521 & 4.058711 & 3 & 2 & 658009 & 614712 \\
0,8 & 4.032938 & 4.092391 & 2 & 1 & 878968 & 263980 \\
0,9 & 4.076117 & 4.064959 & 2 & 1 & 808981 & 221234 \\
\hline
\end{tabular}

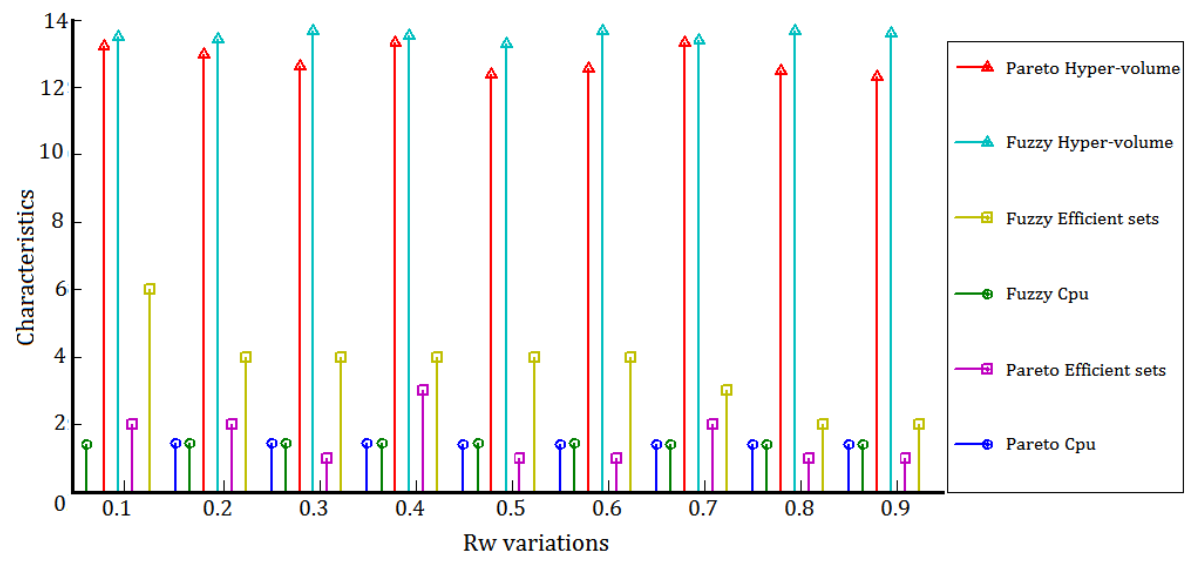

Figure 2. Random walk influence.

Table 2 presents the influence of random walk on the Tabu search method, This is achieved by varying the parameter $W$ by 0.1 at each step on the range $[0.1,0.9]$ for a fixed number of steps (200). The instance Ca2-50-8 of the $\mathrm{WDP}_{t}$ is selected for this test. For each run, the sizes of the effective solutions are recorded. The obtained results are presented in Table 2 and illustrated in Figure 2.

Table 3 contains the results of the influences between the number of iterations and the running time, efficient set and hypervolume, thus, influences between, Hypervolume/Efficient sets, Cpu time/Efficient sets and Cpu time/Hypervolume. The problem test ca50-8 was also selected for this study.

Results of influences parameters and comparison between both mechanisms are depicted by Figure 3 .

After reviewing the results of these implementations, we note that:

- The threshold of the random walk influences directly the size of efficient set, therefore hypervolume.

- The cpu time, hypervolume and efficient set size increase proportionally with the number of iterations increasing.

- The hypervolume output from each execution is proportional to the size of the efficient set.

Table 4 contains the results of implementation on machine. Size of instances problem (B-CAP) varies between 3 and 12 constraints and 5-500 variables for a fixed value of random walk 0,3 . To address the heuristic nature of the Tabu method, multiple runs for each mechanism are made, then the best result is chosen. The two sets 
TABLE 3. Influences (number of steps, cpu time, hypervolume, efficient set).

\begin{tabular}{llccl}
\hline \hline Number of steps & Fuzzy/Pareto & Cpu time & Efficient set size & Hypervolume \\
\hline \multirow{5}{*}{50} & Pareto TS & 1.087582 & 1 & 219392 \\
& Fuzzy TS & 1.184999 & 2 & 579561 \\
100 & Pareto TS & 2.152525 & 2 & 795668 \\
& Fuzzy TS & 2.311846 & 4 & 838457 \\
150 & Pareto TS & 3.170974 & 2 & 570505 \\
& Fuzzy TS & 3.240528 & 4 & 636415 \\
200 & Pareto TS & 4.347353 & 1 & 261300 \\
& Fuzzy TS & 4.226346 & 3 & 446406 \\
250 & Pareto TS & 5.476409 & 1 & 258180 \\
& Fuzzy TS & 5.535162 & 3 & 888463 \\
300 & Pareto TS & 6.296518 & 1 & 246132 \\
& Fuzzy TS & 6.437656 & 4 & 748126 \\
350 & Pareto TS & 7.096277 & 4 & 509700 \\
& Fuzzy TS & 7.096271 & 7 & 744711 \\
400 & Pareto TS & 8.405027 & 1 & 268268 \\
& Fuzzy TS & 8.523825 & 2 & 644447 \\
450 & Pareto TS & 9.804638 & 1 & 654001 \\
& Fuzzy TS & 9.504429 & 2 & 293664 \\
500 & Pareto TS & 10.113463 & 2 & 715776 \\
& Fuzzy TS & 10.339936 & 4 & 733502 \\
\hline
\end{tabular}

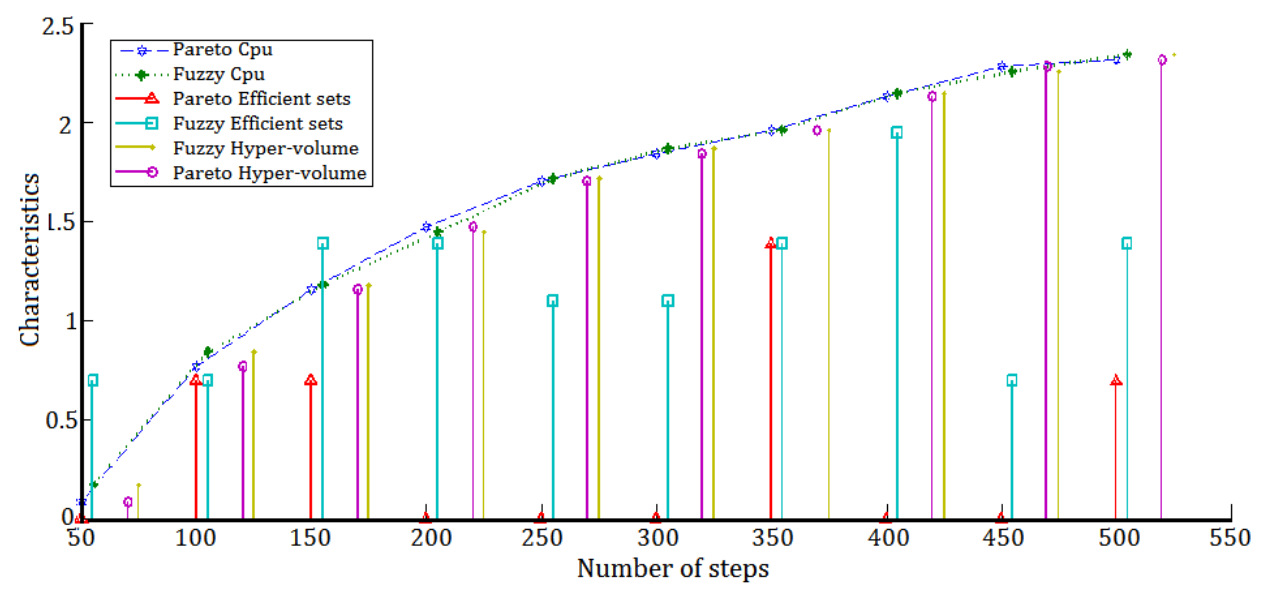

FiguRE 3. Number of steps influences.

of efficient solutions obtained by the two mechanisms will be tested and will be compared by the hypervolume metric.

Figure 4 gives rise to a comparison of the two methods by the hypervolume ticket, that is a performance index used to compare the volumes generated by the sets of efficient solutions. Recall here that the comparison criterion is proportionally linked to the type of objective functions. Indeed hypervolume metric is better than any another one in our case (maximization) if and only if it is higher. 
TABLE 4. Computation results.

\begin{tabular}{|c|c|c|c|c|c|c|c|}
\hline \multirow{2}{*}{$\begin{array}{c}\text { Time/Metrics } \\
\text { instances }\end{array}$} & \multirow{2}{*}{$\begin{array}{l}\text { Number } \\
\text { of steps }\end{array}$} & \multicolumn{2}{|c|}{ Time resolution } & \multicolumn{2}{|c|}{ Hypervolume } & \multicolumn{2}{|c|}{ Efficient set size } \\
\hline & & $\begin{array}{l}\text { Fuzzy } \\
\text { TS }\end{array}$ & $\begin{array}{c}\text { Pareto } \\
\text { TS }\end{array}$ & $\begin{array}{l}\text { Fuzzy } \\
\text { TS }\end{array}$ & $\begin{array}{c}\text { Pareto } \\
\text { TS }\end{array}$ & $\begin{array}{c}\text { Fuzzy } \\
\text { TS }\end{array}$ & $\begin{array}{c}\text { Pareto } \\
\text { TS }\end{array}$ \\
\hline $2 \mathrm{ca} 5-2$ & 20 & 0.497525 & 0.515229 & 2783 & 2783 & 1 & 1 \\
\hline 2 ca5-3 & 20 & 0.498827 & 0.500535 & 5936 & 5936 & 1 & 1 \\
\hline 2 ca5-4 & 20 & 0.528300 & 0.476851 & 43014 & 43014 & 2 & 2 \\
\hline $2 \mathrm{ca} 8-2$ & 50 & 1.145255 & 1.130748 & 56416 & 39791 & 4 & 3 \\
\hline $2 \mathrm{ca} 8-4$ & 50 & 1.182007 & 1.191154 & 33200 & 31501 & 2 & 2 \\
\hline $2 \mathrm{ca} 8-5$ & 50 & 1.126479 & 1.060983 & 36280 & 35185 & 3 & 2 \\
\hline 2ca10-2 & 70 & 1.574336 & 1.481346 & 55752 & 49189 & 3 & 4 \\
\hline 2ca10-4 & 70 & 1.570095 & 1.763708 & 54868 & 15456 & 3 & 1 \\
\hline 2ca10-5 & 70 & 1.742172 & 1.670500 & 58836 & 36629 & 2 & 3 \\
\hline 2 ca20-4 & 100 & 2.376816 & 2.304323 & 1924857 & 1587861 & 6 & 3 \\
\hline 2 ca20-5 & 100 & 2.394490 & 2.453457 & 5174121 & 3325273 & 2 & 2 \\
\hline 2 ca20-7 & 100 & 2.400410 & 2.378088 & 283282 & 255183 & 3 & 2 \\
\hline 2ca30-5 & 140 & 3.578758 & 3.669310 & 934041 & 220890 & 2 & 1 \\
\hline 2ca30-6 & 140 & 3.648789 & 3.514757 & 615184 & 576925 & 4 & 2 \\
\hline 2ca30-9 & 140 & 3.323080 & 3.362119 & 419895 & 369745 & 1 & 1 \\
\hline $2 \mathrm{ca} 40-8$ & 200 & 4.826474 & 5.044656 & 543665 & 516560 & 4 & 4 \\
\hline 2ca40-10 & 200 & 4.804330 & 4.861535 & 501430 & 306574 & 3 & 6 \\
\hline 2ca40-12 & 200 & 5.204353 & 4.976486 & 643651 & 525822 & 5 & 2 \\
\hline 2 ca50-4 & 250 & 6.395765 & 6.673607 & 525252 & 499410 & 1 & 1 \\
\hline 2 ca50-6 & 250 & 5.851583 & 5.732624 & 1249696 & 430612 & 3 & 1 \\
\hline 2 ca50-8 & 250 & 5.842883 & 5.930319 & 737242 & 724584 & 4 & 1 \\
\hline 2 ca80-3 & 300 & 7.145285 & 7.251330 & 1431490 & 1428960 & 1 & 1 \\
\hline 2 ca80-6 & 300 & 7.025340 & 7.013142 & 5663436 & 2274098 & 1 & 1 \\
\hline 2 ca $80-8$ & 300 & 6.982457 & 7.117022 & 2700390 & 2430981 & 1 & 1 \\
\hline 2 ca100-3 & 500 & 12.183902 & 12.092765 & 578547 & 504643 & 2 & 1 \\
\hline 2 ca100-4 & 600 & 14.532469 & 14.582653 & 228045 & 219487 & 3 & 2 \\
\hline 2 ca100-8 & 500 & 14.658570 & 15.050283 & 1316520 & 975125 & 2 & 1 \\
\hline 2 ca200-4 & 900 & 22.591620 & 22.771622 & $1.439 \cdot 10^{9}$ & 113039791 & 6 & 1 \\
\hline 2 ca200-5 & 900 & 22.371261 & 22.388491 & 133921324 & 122955240 & 2 & 3 \\
\hline 2 ca200-7 & 800 & 18.297821 & 18.442591 & 105233679 & 15712825 & 4 & 2 \\
\hline 2 ca500-2 & 1500 & 31.150984 & 31.124524 & 11653664 & 6164795 & 5 & 2 \\
\hline 2 ca500-3 & 1500 & 30.942580 & 31.213084 & 1808989 & 2117837 & 9 & 5 \\
\hline 2 ca500-5 & 1500 & 31.168190 & 31.462098 & 8010090 & 7468070 & 4 & 3 \\
\hline
\end{tabular}

\subsection{Result analysis}

The numerical experiments performed on all benchmarks give rise to interesting remarks, emphasizing the effectiveness of fuzzy comparison mechanism. Thus, it is obvious that the cpu time is not the only performance index to evaluate on a multi-objective resolution process. In this part we also compare the results obtained through hypervolume and size of efficient set.

In all tested problems we notice an approximation of the cpu time between the two mechanisms with a slight superiority of fuzzy comparison mechanism. However, a net superiority of fuzzy mechanism comparison in terms of hypervolume and efficient set size is observed for almost the tested benchmark.

However, the Tabu search with Fuzzy dominance get the top scores for the majority of the issues tested. Except for some instances, where the cpu time is less important, the quality of generated Pareto front is also consistent. And also in general the cpu time increases proportionally with the problem size and number of iterations. 


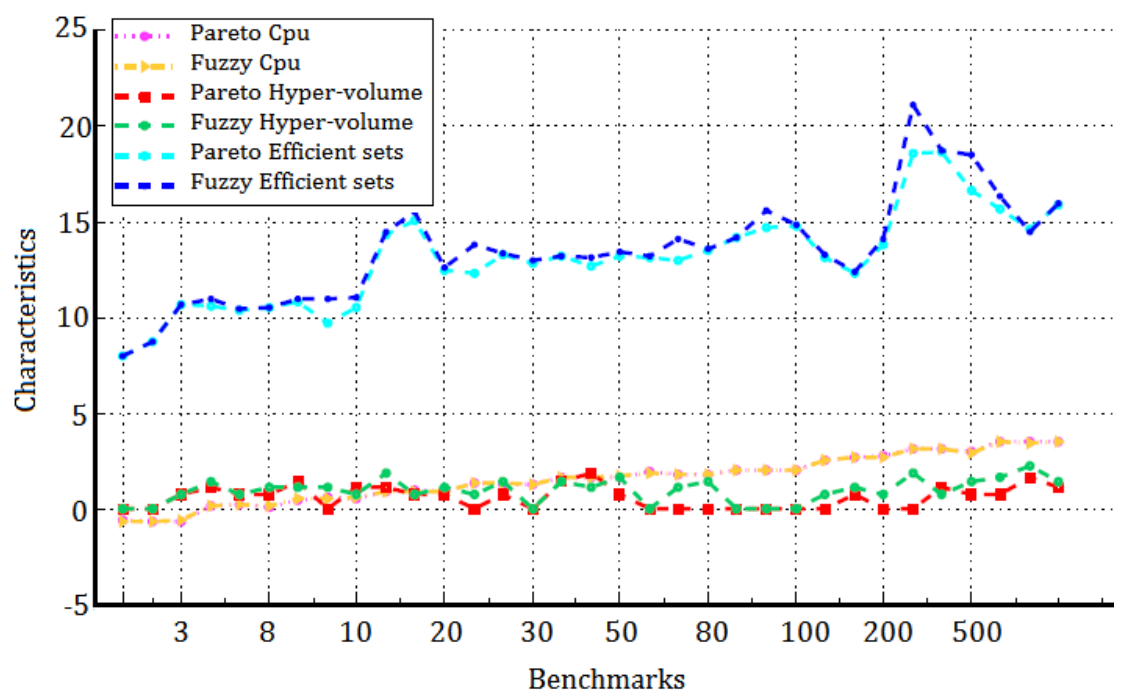

FiguRE 4. Performance index.

Hypervolumes obtained by fuzzy process are significantly higher for each problem instance, although we note, despite that, for some cases the sizes of Pareto fronts obtained based on Pareto dominance are more important than those obtained by the fuzzy process, but the corresponding hypervolumes are less important. This shows that the number of solutions can not be in itself a determinant factor for the quality of the Pareto front, but must be associated with other performance measures.

\section{CONCLUSion AND FUture WORKS}

In this work, we studied the problem of combinatorial auctions CAP "Dynamic winner determination problem". For this, we have developed a new "bi-objective" model based on the English auction mechanism, that is built gradually as bidders launch their offers during the exercise period. Then an algorithm hybridizing tabu search method with the comparison mechanism solutions based on fuzzy logic was designed, which gives the list of winners at any time of the exercise period.

A numerical study was carried out by implementing the mechanisms developed on machine and a set of different sizes of test problems is designed and tested, a comparison between the fuzzy and Pareto mechanisms is performed based on the hypervolume metric. The results show that the fuzzy mechanism is promising.

For future works, it is very interesting to generalize the developed model by giving more freedom to bidders when updating their offers, on the one hand, and try to make an approach based on an hybridization of an exact method and fuzzy dominance on the other hand.

\section{REFERENCES}

[1] A. Anderson, M. Tenhunen and F. Ygge, Integer programming for combinatorial auctions winner determination. In: Proc. of 4th International Conference on Multi-Agent Systems. IEEE Computer Society Press (2000) 39-46.

[2] D. Boughaci, B. Benhamou and H. Drias, Memetic Algorithms for the Optimal Winner Determination Problems in Combinatorial Auctions. J. Soft Comput., 13 (2009) 905-917.

[3] T. Buer and H. Kopfer, A Pareto-metaheuristic for a bi-objective winner determination problem in a combinatorial reverse auction. Comput. Oper. Res., 41 (2014) 208-220.

[4] D. Chakraborty, D. Guha and B. Dutta, Multi-objective optimization problem under fuzzy rule constraints using particle swarm optimization. Soft Comput. 20 (2016) 2245-2259.

[5] M. Ehrgott, Multicriteria Optimization, 2nd edition. Springer, Berlin Heidelberg New York (2005). 
[6] S. Ganguly, N.C. Sahoo and D. Das, Multi-objective particle swarm optimization based on fuzzy-Pareto-dominance for possibilistic planning of electrical distribution systems incorporating distributed generation. Fuzzy Sets Syst. 213 (2013) 47-73.

[7] R. Garg and A-K. Singh, Multi-objective workflow grid scheduling using $\varepsilon$-fuzzy dominance sort based discrete particle swarm optimization. J. Supercomput. 68 (2014) 709-732.

[8] Y. Guo, A. Lim, B. Rodrigues and Y. Zhu, Heuristics for a bidding problem. Comput. Oper. Res. 23 (2006) 2179-2188

[9] A. Holland and B. O'sullivan, Towards fast Vickrey pricing using constraint programming. Artif. Intell. Rev. 21 (2004) 335-352.

[10] N.E.A. Khalid, N.A. Bakar, F.Sh. Ismail and N.S.M. Dout, Multi-objective optimization using fuzzy evolutionary strategies optimization. Int. J. Syst. Appl. Eng. Dev. 5(6) (2011) 728-737.

[11] M. Köppen, R. Vicente-Garcia, and B. Nickolay, Fuzzy-pareto-dominance and its application in evolutionary multi-objective optimization. Lect. Notes Comput. Sci. 3410 (2005) 399-412

[12] R.L. Leskelä, Bidder Support In Iterative Multiple-Unit Combinatorial Auctions. Ph.D. thesis. Helsinki University of Technology, Finland (2009).

[13] J. Levin and A. Skrzypacz, Are dynamic Vickrey auctions practical? Properties of the combinatorial clock auction. Economic Policy Research Discussion Paper. Stanford Institute 14-002 (2014).

[14] K. Leyton-Brown, M. Tennenholtz and Y. Shoham, An algorithm for multi-unit combinatorial auctions. In: Proc. of the 17th National Conference on Artificial Intelligence and Twelfth Conference on Innovative Applications of Artificial Intelligence (2000) 56-61.

[15] N. Nisan, Bidding and allocation in combinatorial auctions. In: Proceedings of the ACM Conference on Electronic Commerce (EC-00), Minneapolis. ACM SIGecom, ACM Press (2000) 1-12.

[16] J. Razmia, E. Jafarian and S. Hassanzadeh-Amin, An intuitionistic fuzzy goal programming approach for finding pareto-optimal solutions to multi-objective programming problems. Expert Syst. Appl. 65 (2016) 181-193.

[17] R. Saborido, Ana B.Ruiz, J. D.Bermdez, E. Vercher and M. Luque, Evolutionary multi-objective optimization algorithms for fuzzy portfolio selection. Appl. Soft Comput. 39 (2016) 48-63.

[18] N.C. Sahoo, S. Ganguly and D. Das, Fuzzy-Pareto-dominance driven possibilistic model based planning of electrical distribution systems using multi-objective particle swarm optimization. J. Expert Syst. Appl. 39 (2012) 881-893.

[19] M. Sakawa,K. Katoa, H. Sunadaa and T. Shibanob, Fuzzy programming for multiobjective 0-1 programming problems through revised genetic algorithms. Eur. J. Oper. Res. 97 (1997) 149-158.

[20] T. Sandholm, S. Suri, A. Gilpin and D. Levine, CABOB; A fast optimal algorithm for winner determination in combinatorial auctions. Manag. Sci. 51 (2005) 374-390.

[21] P. Sariddichainunta and K. Sinapiromsaran, The winner determination model and computation for linear arrangement of booth auction. Inf. Technol. J. 7 (2011) 46-51.

[22] E. Zitzler, Evolutionary algorithms for multiobjective optimization: methods and applications. Ph.D. thesis, Swiss Federal Institute of Technology (ETH), Zurich, Switzerland (1999). 